\title{
Current Concepts in Rectal Cancer
}

\author{
James W. Fleshman, MD, FACS, FASCRS ${ }^{1}$ Nathan Smallwood, MD ${ }^{1}$ \\ ${ }^{1}$ Department of Surgery, Baylor University Medical Center at Dallas, \\ Dallas, Texas \\ Address for correspondence James W. Fleshman, MD, FACS, FASCRS, \\ Department of Surgery, Baylor University Medical Center at Dallas, \\ 3500 Junius St, Dallas, TX 75246 \\ Clin Colon Rectal Surg 2015;28:5-11. \\ (e-mail: James.Fleshman@baylorhealth.edu).
}
Abstract
Keywords
- colon and rectum
- colorectal disease
- neoadjuvant therapy
- multidisciplinary

The history of rectal cancer management informs current therapy and points us in the direction of future improvements. Multidisciplinary team management of rectal cancer will move us to personalized treatment for individuals with rectal cancer in all stages.
Since the first recorded surgical resection for rectal cancer in 1826 by Jacques Lisfranc, ${ }^{1}$ the management of rectal cancer has evolved remarkably with dramatic improvements in local recurrence and mortality. Surgical resection is the only proven curative treatment of rectal cancer and the important advances in care have involved improvements in surgical techniques. Most of these improvements during this time period resulted from a better understanding of the anatomy and pathology of rectal cancer. It is important to be familiar with these important advances in rectal cancer treatment to fully understand the current methods of treatment.

\section{Historical Overview: From Miles' Abdominoperineal Resection to Today's Sphincter-Preserving Techniques}

\section{Rectal Resections before Miles}

Giovanni Morgagni in the early 18th century was the first to propose rectal resection as a treatment for cancer. ${ }^{1}$ However, it took over a century before the first successful rectal resection was performed. Over the years, there have been constant changes in the approach used by surgeons to perform a rectal resection.

Rectal resections were first performed using a perineal approach as described in 1826 by Jacques LisFranc. ${ }^{1,2} \mathrm{He}$ operated in the era before anesthesia and proper antiseptic techniques. Since inadvertent entry into the peritoneal cavity could prove lethal, he chose the perineal approach to evert the rectum and perform a limited resection below the peritoneal reflection. A limited amount of rectum was resected with this approach and no attempt was made to purposely include the mesorectum and draining lymph nodes. ${ }^{2}$ Success at that time was based upon whether the patient survived to leave the hospital, and so the risk of local recurrence had very little influence. With the advent of anesthesia and aseptic techniques, operations could now be performed that would provide for a more radical resection. Paul Kraske developed a technique similar to Kocher's technique, where he would incise and detach the left side of the coccyx and sacrum to provide more exposure. This exposure allowed him to resect the rectal cancer with $1 / 2$ inch margins on either side of the rectum. The proximal bowel would then be pulled down and sutured to the anal sphincter complex. However, this "sacral anus" was difficult for the patient to manage. ${ }^{1,3}$

Carl Guessenbauer performed the first transabdominal rectal resection and with closure and colostomy in 1879 . This procedure would be later popularized by Henri Hartmann for the purpose of treating diverticulitis. ${ }^{4}$ Vincent Czerny is credited with performing the first combined abdominal and perineal approach. This combined approach was never intended but was performed only after an unsuccessful attempt at resection through the perineal approach. ${ }^{1}$ In Vogel's review of 1,500 cases performed by 12 of the most prominent 19th century surgeons including Billroth, Kocher, Kraske, and Czerny there was a $21 \%$ operative mortality rate with a high (80\%) recurrence rate. ${ }^{3}$ Surgery certainly had progressed beyond LisFranc's perineal resections; however, the results were merely palliative in the vast majority of cases.

\section{Sir William Ernest Miles and the Battle to Prevent Local Recurrence}

The improvement in mortality for the surgical treatment of rectal cancer from 100 to $4 \%$ over the past 250 years is also due to a great many advancements in medicine. ${ }^{1}$ None has
Issue Theme Rectal Cancer; Guest Editors: James W. Fleshman, MD, FACS, FASCRS, and Warren E. Lichliter, MD, FACS, FASCRS
Copyright (c) 2015 by Thieme Medical Publishers, Inc., 333 Seventh Avenue, New York, NY 10001, USA.

Tel: +1(212) 584-4662.
DOI http://dx.doi.org/ 10.1055/s-0035-1545064. ISSN 1531-0043. 
been more important than the growing knowledge of rectal anatomy and sophisticated pathology evaluating tumor spread and lymph node involvement. Sir William Ernest Miles can be credited with the first to emphasize the importance of these factors in preventing local recurrence and reducing mortality. Unfortunately, the knowledge he gained in these areas came at the expense of his surgical failures. Like all of his predecessors and surgeons of his era, Miles would witness recurrence of cancer following perineal resection. Frustrated by these failures, Miles exhaustively studied the results of his operations from 1899 to 1906. He observed the clinical natural history in inoperable patients and performed extensive post mortem dissections on these and his own patients. Through all of this, he hoped to gain a better understanding of the usual routes by which rectal cancer spread. ${ }^{1,5} \mathrm{He}$ first observed recurrences in the ischiorectal fat, leading him to make wider perineal excisions. After this modification, all 14 of his patients had recurrence, but now these recurrences followed a more lateral spread. In the 11 operations to follow, he decided to excise the rectal fascia propria and mesorectum, along with the levator ani muscles and opening up the peritoneum to excise another $3 \mathrm{~cm}$ of proximal bowel. All 11 had recurrence, with the majority involving the mesenteric tissues more proximal and intra-abdominal to his dissection. Further attempts to excise the entire mesorectum proved impossible with the majority of recurrences occurring in the pelvic mesocolon. Through all of these modifications, Miles doubled the time before recurrence, but a $100 \%$ recurrence rate mandated a new approach. ${ }^{1,2,5}$

By now Miles had distinguished, based upon his observations, three zones of spread. With his own modifications in technique, he was able to successfully eliminate both the downward and lateral spread of rectal cancer. However, he knew from his own operations that the zone of upward spread could not be eradicated through the perineal approach. This would lead him to first performing an abdominal midline approach followed by a perineal resection. He was not the first to combine these approaches and he himself acknowledged such contributors in his landmark article. He would, however, further clarify that these prior attempts had failed to eradicate the zones of upward spread. ${ }^{5}$ Like Czerny and surgeons after him, an abdominal approach was mainly used to provide more mobility to the rectum to facilitate the rectal resection through a perineal approach. Miles used this abdominal approach to gain better access to and eradicate his perceived zone of upward spread. Specifically, the areas involved included the pelvic mesocolon, lymph nodes over the left common iliac artery, and the peritoneum. From his extensive observations in the spread of rectal cancer, he then formulated "certain essentials in the technique of the operation." 5

- The abdominal anus is a necessity.

- The whole of the pelvic colon must be removed because the zone of upward spread involves its blood supply.

- The whole of the pelvic mesocolon below the point where it crosses the common iliac artery, together with a strip of peritoneum at least an inch wide, on either side must be cleared.

- The lymph nodes around the bifurcation of the common iliac artery are to be removed in all instances.

- The perineal portion of the operation must be performed as widely as possible to fully eradicated both lateral and downward zones of spread.

In his landmark article, Miles revealed the results of his first 12 operations for which he called an abdominoperineal excision. He acknowledged that the $41.6 \%$ mortality rate was indeed too high and that with further experience he could decrease this rate. Overtime, Miles would be able to decrease the rate of recurrence to $29.5 \%$, which was a monumental achievement when compared with the usual rates of recurrence at that time. ${ }^{1}$ Miles' abdominoperineal excision would eventually become the standard procedure in the treatment of rectal cancer, but only after further refinements in technique, in addition to advances in anesthesia and blood transfusions.

Surgeons now began to recognize the importance of the upward spread of rectal cancer. Some, like Lockhart-Mummery, recognized this pattern of spread but believed that actual cancer involvement of these nodes indicated incurability. ${ }^{6}$ Both Miles and Lord Moynihan emphasized this pattern of spread and each advocated for proximal ligation of the inferior mesenteric artery. Unlike Miles, Moynihan, however, believed the best point of ligation was even higher and should be done proximal the left colic branch. This debate continues to this day. ${ }^{7}$

\section{Birth of Sphincter-Sparing Techniques}

\section{Miles' Major Error}

The abdominoperineal resection (APR) as described by Miles was recommended for all rectal cancers including those above the peritoneal reflection, which are currently treated with sphincter-sparing low anterior resections (LARs) of the rectosigmoid. This belief was predicated on Miles' erroneous assumption that these more anterior rectal tumors could spread in a downward direction. Since the APR was the gold standard of the day, this theory would have to be disproven before colostomies could be prevented and bowel continuity restored.

While some surgeons questioned the morbidity involved in performing the Miles' APR, others began to question its need for all rectal cancers. ${ }^{1}$ Cuthbert Dukes, a pathologist at St. Marks Hospital in London, well known for his classification system of rectal cancer, saw a disparity between his clinical observations and that of Miles. He often saw lymph node spread parallel and proximal to the tumor and felt that downward spread in rectal cancer had been overemphasized. Other pathologic studies during the 1930s likewise contradicted Miles. There now became a shift toward anterior resection and anastomosis for mid and proximal rectal cancers. $^{7}$

Unfortunately, the mortality rate was high for anterior resections, as first described by Balfour, secondary to leaks 
from the anastomosis. ${ }^{1,7}$ Claude Dixon, in 1948, was the first to prove that anterior resections could be safely done. He presented to the American Surgical Association his results from some 426 anterior resections and showed that a very low $2.6 \%$ mortality rate could be achieved. ${ }^{1}$ Further, he proved with his results that such resection was oncologically sound. ${ }^{2}$ In 1970, Sir Alan Parks, at Saint Marks Hospital, showed that rectal cancers even closer to the dentate line cutoff could be safely resected and a coloanal anastomosis performed. He achieved comparable results for cancers treated with APR. All of Dixon's resections involved cancers $6 \mathrm{~cm}$ or more from the dentate line. ${ }^{1}$ Sphincter-sparing operations became the goal once the operation was proved to have comparable oncological outcomes as the gold standard of APRs. Progress continues toward this goal.

\section{Challenging the Distal Margin}

One of the major concerns in sphincter preservation is the ideal distal margin. The initial $5 \mathrm{~cm}$ "safe margin" as set forth by Goligher, Dukes, and Bussey was a severe limitation for the surgeon treating a patient with a low rectal tumor. ${ }^{7}$ Even during its initial adoption, the $5 \mathrm{~cm}$ rule was constantly being challenged. Not until the 1990s was this changed to the $2 \mathrm{~cm}$ rule. Currently, in the setting of neoadjuvant chemoradiation, even this $2 \mathrm{~cm}$ margin has been challenged to require only a negative margin on the pathologic specimen. Those tumors that are in the distal $3 \mathrm{~cm}$ of rectum require close margins to spare the sphincter and transition zone of the anal canal. ${ }^{7}$ Surgeons are attempting to resect even lower tumors with subsequent reconstruction. There remains a need to improve and create new techniques to meet these new technical demands.

\section{Technical Improvements}

The major challenges of rectal cancer surgery are due to its location within the bony confines of the pelvis. These challenges increase significantly in morbidly obese patients, male patients with a narrow pelvis, in some patients with prior chemoradiation, and in large, locally advanced cancers. Sphincter preservation is less likely in the larger but completely resectable tumors. Current data support the belief that surgeon volume, and presumably skill, is one of the two most important factors in sphincter preservation. ${ }^{8,9}$

While technical ability has always varied among surgeons, technology has usually been able to help equalize this variation. For rectal cancers, the surgical stapler was the device which helped surgeons work more efficiently in this technically demanding location, facilitating safe anastomoses and even lower resections. Staplers were first used by Humer Hultl in 1908 for a gastrectomy. ${ }^{1}$ Fifty years later, Mark Ravitch brought back the design of earliest Russian staplers and, following a few modifications, began using them in the United States. ${ }^{1}$ The most important of all staplers for the colorectal surgeon, the end-to-end anastomosis (EEA) stapler, was first successfully used in $1977 .{ }^{10}$ Until 1977, the low anastomosis was performed much like a cardiac valve procedure by "parachuting" a hand sewn end-to-end suture line into the deep pelvis. The EEA stapler allowed for more efficient and technically sound anastomosis in the low pelvis. The widespread use of the EEA stapler in the late 1970s and 1980s significantly reduced the need for APR. ${ }^{10}$

Laparoscopy has provided to colorectal surgery its wellknown benefits, such as decrease length of stay and better pain control. However, the recovery of bowel function and anastomotic healing is more influential on length of stay than reduction in abdominal incision length as seen in other operations. The equivalency of oncologic outcomes between laparoscopic and open colon resections has been proven in multiple randomized control trials. The equivalencies of laparoscopic and open treatment of rectal cancers have been an ongoing debate, and currently randomized trials are in progress. The one overwhelming advantage when using laparoscopy is the improved visualization during dissection. R. J. Heald, the major influence in the adoption of the total mesorectal excision (TME), has likewise acknowledged the superior visualization when using the laparoscope. TME requires dissection in an areolar tissue plane in the pelvis outside the mesorectal fascia. However, the laparoscopic technique within the pelvis is limiting due to the angles of approach and linear effector positions.

Robotic surgery has the possibility to extend this further, not only by improving the visualization during surgery but also by potentially overcoming some of the technical limitations that exist when performing laparoscopy in the pelvis. Improved retraction and the advantage of wristed or articulating instruments facilitate dissection within the narrow pelvis. Indeed, the literature has shown evidence of its merits for rectal resections in the male pelvis as well as mid- and low-rectal tumors. ${ }^{11-13}$ Whether any of these benefits will outweigh the cost of robotic assisted surgery will be addressed in the RObotic versus LAparoscopic Resection for Rectal cancer (ROLARR) study.

\section{Neoadjuvant Therapy}

In the era before the introduction of TME, surgical resections which did not focus on maintaining the mesorectal envelope resulted in a 15 to $45 \%$ rate of local recurrence. Even though surgical techniques had advanced substantially, these rates of recurrence proved there was a need for additional recurrence-lowering therapies. Radiation to the pelvis to treat locally advanced rectal cancers began in 1914 . $^{1}$ Based upon trials in the 1980s, 5FU-based postoperative chemoradiation became the standard of care up to the 1990 s. ${ }^{14}$ Postoperative chemoradiotherapy was seen to significantly improve the low recurrence rates achieved with TME. Despite the improvement in recurrence rates, postoperative chemotherapy was associated with high toxicity rates and poor functional outcome after sphincter-sparing surgery. In an effort to improve the tolerability of chemoradiation, multiple clinical trials were performed testing preoperative with postoperative administration. Trials like the German Rectal Cancer Study Group showed that improved local control and reduced toxicity could be achieved when chemoradiation was given preoperatively. ${ }^{1}$ Additional studies showed that the addition of neoadjuvant chemoradiation to TME could reduce the rate of local recurrence from 8.2 to $2.4 \%{ }^{14}$ 
Currently, neoadjuvant chemoradiation is believed to improve the rate of sphincter preservation in two ways. First, as the tumors are downsized by a response to chemoradiation, the tumor becomes smaller and more manageable with less collateral tissue resection, which may convert the patient to an LAR instead of an APR. Second, for patients whose tumors have had a complete pathologic response, the option for local excisions or observation exists, but is currently under investigation. Unfortunately, most large studies have been unable to show any significant increase in the rate of sphincter preservation with the use of neoadjuvant therapy despite clearly showing its oncological advantages. ${ }^{15,16}$ These results are still not clear when one looks at whether short or long course therapies were given or how long of an interval to wait. $^{16}$ The use of local excision offers the potential for sphincter preservation, but it is still being explored.

The skilled surgeon is ultimately much more likely to have increased rates of sphincter preservation. This fact is exemplified by Heald's $89.6 \%$ rate of sphincter preservation, far surpassing even specialized centers. In this highly selected group of people, only $9 \%$ of this group received preoperative radiotherapy. ${ }^{17}$ In Europe and Scandinavian countries, it was not until after education-based workshops that the rates of TME and sphincter preservation significantly improve. ${ }^{18}$ At present, chemoradiation should mainly be considered for its improvement in the rate of local recurrence and tumor downstaging, but in the future better knowledge of tumor biology may improve the oncological success rates for techniques of local excision.

\section{Sphincter-Sparing Techniques for Distal Tumors}

Reported rates of sphincter-sparing resections among colon and rectal specialist have been reported as high as 70 to $90 \%{ }^{9}$ Nationally, only about one out of every two patients will be given a sphincter-sparing operation. ${ }^{19}$ Even with the ability for neoadjuvant therapies to provide tumor downsizing, tumors located in the distal one-third of the rectum continue to challenge our ability to provide both an optimal oncological resection and a sphincter-sparing operation. These challenges are mainly due to funnel shape of the pelvis anatomy. It becomes increasingly more difficult to adequately resect the cylindrical piece of tissue consisting of the rectum and mesorectum from the progressively narrowing funnelshaped pelvis. This is why obtaining adequate lateral margins is often much more difficult than to obtain clear distal margins. The challenges of the pelvic anatomy are even worse in patients who have a large amount of intra-abdominal and pelvic fat resulting in positive margins and noncurative resections.

The introduction of the EEA stapler in 1979 and the universal adoption of the double stapling technique, first described by Knight and Griffin in 1980, overcame some of the technical challenges in performing LARs and certainly facilitated the progress toward performing more sphinctersparing operations. The conventional double stapling technique is much less helpful, however, in attaining an adequate distal resection margin for tumors located in the distal onethird of the rectum. The narrowness of the low pelvis makes placement of a linear stapler difficult even in an open operation. Laparoscopic endostaplers are more likely to produce tangential resection line and require multiple staple lines. This may result in positive distal margins or the risk of anastomotic leak, respectively. ${ }^{20,21}$

Technical challenges that are faced when resecting these very low rectal cancers has stimulated surgeons to invent an array of new techniques which overcome the inadequacies of the existing conventional methods to overcome these challenges. These new techniques, however, have only been able to be utilized by the more highly trained and specialized surgeons, and are still an emerging field of Colon and Rectal surgery. In the United States, most rectal cancers are resected by surgeons experienced only in the conventional open or laparoscopic methods that are more suited for more proximal cancers.

The first methods created to combat low rectal tumors were pull through procedures first described by Maunsell in 1892 and later described by Cutait and Turnbull in the 1960s and 1970s. These operations removed the entire dentate line and pulled the colon through the anus to allow the fusion of the bowel to the anal canal. The redundant colon, which became gangrenous, was then amputated at the anal verge 7 days later. Sir Alan Park later refined the pull-through into performing just a coloanal anastomosis in $1972 .{ }^{22}$ Later, surgeons would begin to exploit the natural plain between the internal and external sphincter muscles to begin the dissection before excising the rectum along with some or all of the internal sphincter. This procedure would be further refined by Dr. Gerald Marks in 1982 and given the name transabdominal transanal proctosigmoidectomy or TATA. ${ }^{23}$

Sphincter-preserving techniques such as the intersphincteric resection were created to better delineate the distal margin and allow for an easier resection. ${ }^{24}$ These bottom-up dissections continue to be extended cephalad further and further owing to the ease with which the mesorectum can be visualized and resected in the narrow male pelvis. This in-line visualization of the pelvic structures aids in a better quality mesorectal resection overall, particularly in the distal most pelvis. ${ }^{24}$ A different array of platforms such as the transanal endoscopic microsurgery (TEM) and transanal minimally invasive surgery (TAMIS) have allowed for improved visualization through endoscopy and use of longer instruments passed through the anal canal opening.

\section{Local Therapy}

Due to the historically high rates of local recurrence in rectal cancer, transanal excision without radical resection was only rarely considered. Recently, three factors have resulted in an increase in the overall interest in not only sphincter preservation but also organ-preserving treatments. First, despite improvements in technique and postoperative care, rectal resections with TME are associated with significant postoperative morbidity. Second, the rates of tumor downstaging and even complete pathological response following neoadjuvant therapy have brought to question the utility of performing a resection of the mesorectum when there are no viable cells found in the tumor. Third, new technologies such as TEM 
and TAMIS have made local resections technically more feasible.

Currently, the National Comprehensive Cancer Center Network Guidelines state that candidates for full thickness local resection include Tis and T1 tumors up to $3 \mathrm{~cm}$ in size which are well to moderately differentiated, less than onethird the circumference of the rectal lumen and within $8 \mathrm{~cm}$ from the anal verge. Any local resection that results in a final margin less than $1 \mathrm{~mm}$, or cancers revealing lymphovascular invasion, poor differentiation, or occupy the lower one-third of the submucosa requires a more radical resection.

One of the main issues with local resection is that even small lesions, up to T2, can have a $19 \%$ lymph node positivity. ${ }^{25}$ Determination of which tumors (based upon depth, differentiation, or imaging characteristics) are likely to have lymph node involvement has yet to be truly defined. Most recently, excellent staging has been achieved through imaging of the pelvis with a specific type of MR that uses body surface coil phased array scanning and processing of images to provide cross-section images of the rectum. Prior studies have shown that local excision for $\mathrm{T} 1$ lesions has a much higher recurrence rate (13.2 vs. $2.7 \%$ ) than LAR with TME. ${ }^{26} \mathrm{~A}$ recent study using the Surveillance, Epidemiology, and End Results (SEER) database has shown equivalent oncologic outcomes in patients with $\mathrm{T} 1$ cancers as a whole. This same study showed that local excision is safe for downstaged tumors following neoadjuvant chemoradiation, including T2 tumors. $^{27}$ These results are quite improved over the prior studies which showed up to a $15 \%$ recurrence rate. ${ }^{28}$ Certainly, there will need to be improvements in our ability to characterize tumor response to neoadjuvant therapy radiologically before local excision can be routinely recommended.

Endocavitary contact radiation (ECR) or radiotherapy is another technique for treatment of early-stage rectal cancers in selected patients. ECR was first used in 1946 to treat rectal cancer by Lamarque and Gros. ${ }^{29}$ ECR offers an additional form of local treatment of $\mathrm{T} 1$ and possibly $\mathrm{T} 2$ rectal cancers with similarly reported failure rates when compared with local excision. ${ }^{30}$ ECR does not disrupt any anatomical planes and therefore, if it fails, does not inhibit salvage surgery. Disadvantages include lack of a surgical specimen for a full histological examination to better predict the likelihood of failure as is used with local excision. Overall, this form of therapy is not likely to gain any more support in the 21 st century than it did in the 20th century.

\section{Rectal Cancer Today: From Personalizing Our Approach to Standardizing Our Care}

The surgeon has always been at the forefront in the fight to constantly find better methods to improve the outcomes and the rate of sphincter preservation when treating rectal cancer. However, some of the improvements in the treatment of rectal cancer are due to efforts from physicians of other disciplines.

Early in the history of treating rectal cancer, the pathologist began to play an important role, not just in staging the resected tumor, but also in guiding the surgeon on what tissues outside the rectum to include in the resection. Through the observations of the pathologist Cuthbert Dukes, surgeons began to transition from the more radical APRs to the sphincter-sparing LAR. $^{1}$ In 1986, the pathologist Phil Quirke demonstrated the importance of lateral tumor spread in local recurrence. He showed that inadequate resections which lead to positive radial or circumferential resection margins increase the rate of local recurrence. ${ }^{7}$ These results would add further emphasis to the importance of a TME previously established in 1982 by British surgeon Bill Heald. ${ }^{7}$ Through their efforts, the TME would become the gold standard technique for low rectal cancers and decrease local recurrence rates from a high of $20 \%$ down to $4 \%{ }^{7}$

Neoadjuvant chemoradiation is now the standard of care and can lower local recurrence rates when combined with TME compared with TME alone. ${ }^{1}$ Improvements in imaging have dramatically increased our ability to clinically stage a tumor prior to surgery or treatment with chemoradiation. An accurate assessment of the pretreatment stage can help individualize treatment and prevent the morbidity associated with chemoradiation in patients with early rectal cancers. With this advanced knowledge and individualized approach, the treatment of rectal cancer has now become a complex decision-making process that requires specialized knowledge from multiple disciplines.

\section{The Multidisciplinary Approach}

Once a disease whose treatment involved only the technical skills of a surgeon, the treatment of rectal cancer now requires the accurate input and assessment from an array of specialties. Unfortunately, many patients have been treated based upon the old U.S. generalist-centered model resulting in variability of care as seen in local recurrence rates, mortality rates, and permanent stoma rates. ${ }^{31}$ To address these discrepancies and improve patient care, many countries, including the United States, have created centers of excellence. Paramount to these centers of excellence is the multidisciplinary team (MDT) which administers the treatment of rectal cancer to each individual patient using standard care pathways. These standard care pathways are based upon five evidenced-based principles of rectal cancer treatment as outlined by the OSTRiCh (Optimizing the Surgical Treatment of Rectal Cancer) group. ${ }^{31}$

1. TME

2. Measuring the quality of surgery or TME through specific pathology assessment techniques

3. Specialist imaging techniques identifying patients at high risk of local recurrence

4. Administering newer and more effective neoadjuvant and adjuvant therapies

5. Using an MDT approach to identify, coordinate, deliver, and monitor the ideal treatment on an individual patient basis.

The ability of the TME to reduce local recurrence has been discussed. This method is easy to teach and, with its widespread use, has been shown to decrease permanent stoma rates, decrease local recurrence, and even improve 5-year 
survival rates in certain population-based studies. ${ }^{31}$ Pathologic assessment of these specimens provides important prognostic factors related to circumferential margin status as well as a quality indicator of the type of resection performed. Pathologic assessment of the quality of resection provides surgeons with direct feedback and allows surgeons to continue to improve and refine their techniques.

MRI is currently the standard for pretreatment imaging of rectal cancer in Europe and will soon become the standard in the United States. The use of MRI within the MDT format has been shown to reduce the incidence of positive circumferential tumor margins. ${ }^{32,33}$ MR volumetric analysis can reliably predict a tumor's clinical response following neoadjuvant therapy and identify patients with low rectal tumors that are amenable to a sphincter-sparing resection. ${ }^{32}$ The use of MRI in the pretreatment assessment of tumors could also allow for a more tailored approach when using neoadjuvant therapy, thereby reducing some of the morbidity associated with rectal cancer treatment. ${ }^{31}$

The MDT consists of surgeons, radiation and medical oncologists, pathologists, and radiologists. These MDTs have been shown to improve clinical decision making and clinical outcomes in rectal cancers. The American College of Surgeons Commission on Cancer has listed the rectal cancer MDT as a key requirement in the treatment of rectal cancer.

\section{Summary}

The treatment of rectal cancer has undergone tremendous improvements since Miles' introduction of the APR in 1908. All of these improvements have led to an increasing number of sphincter-sparing resections performed, lower local recurrence rates, and improved cancer survival. ${ }^{1}$ More and more of these sphincter-sparing resections are now performed using minimally invasive techniques resulting in shorter patient recovery times. With the development of MDTs, these and future improvements can be incorporated into evidencedbased treatment pathways that provide the ideal treatment on an individual patient-by-patient basis.

\section{References}

1 Galler AS, Petrelli NJ, Shakamuri SP. Rectal cancer surgery: a brief history. Surg Oncol 2011;20(4):223-230

2 Ballantyne GH. Theories of carcinogenesis and their impact on surgical treatment of colorectal cancer. A historical review. Dis Colon Rectum 1988;31(7):513-517

3 Graney MJ, Graney CM. Colorectal surgery from antiquity to the modern era. Dis Colon Rectum 1980;23(6):432-441

4 Corman ML. Contributions of eighteenth and nineteenth century French medicine to colon and rectal surgery. Dis Colon Rectum 2000;43(6, Suppl):S1-S29

5 Miles WE. A method of performing abdomino-perineal excision for carcinoma of the rectum and of the terminal portion of the pelvic colon (1908). CA Cancer J Clin 1971;21(6):361-364

6 Lockhart-Mummery JP. Two hundred cases of the rectum treated by perineal excision. Br J Surg 1926;14(53):110-124

7 Lange MM, Rutten HJ, van de Velde CJ. One hundred years of curative surgery for rectal cancer: 1908-2008. Eur J Surg Oncol 2009;35(5):456-463
8 Purves H, Pietrobon R, Hervey S, Guller U, Miller W, Ludwig K. Relationship between surgeon caseload and sphincter preservation in patients with rectal cancer. Dis Colon Rectum 2005;48(2): 195-202, discussion 202-204

9 Paquette IM, Kemp JA, Finlayson SR. Patient and hospital factors associated with use of sphincter-sparing surgery for rectal cancer. Dis Colon Rectum 2010;53(2):115-120

10 Moran BJ. Stapling instruments for intestinal anastomosis in colorectal surgery. Br J Surg 1996;83(7):902-909

11 Trastulli S, Farinella E, Cirocchi R, et al. Robotic resection compared with laparoscopic rectal resection for cancer: systematic review and meta-analysis of short-term outcome. Colorectal Dis 2012; 14(4):e134-e156

12 Memon S, Heriot AG, Murphy DG, Bressel M, Lynch AC. Robotic versus laparoscopic proctectomy for rectal cancer: a meta-analysis. Ann Surg Oncol 2012;19(7):2095-2101

13 Lin S, Jiang HG, Chen ZH, Zhou SY, Liu XS, Yu JR. Meta-analysis of robotic and laparoscopic surgery for treatment of rectal cancer. World J Gastroenterol 2011;17(47):5214-5220

14 Weiser M. Do improvements in surgical technique mean it is time to be more selective in our approach to neoadjuvant radiotherapy for rectal cancer? Current Colorectal Cancer Reports 2014;10(2): 173-179

15 Kapiteijn E, Marijnen CA, Nagtegaal ID, et al; Dutch Colorectal Cancer Group. Preoperative radiotherapy combined with total mesorectal excision for resectable rectal cancer. N Engl J Med 2001;345(9):638-646

16 Baker B, Salameh H, Al-Salman M, Daoud F. How does preoperative radiotherapy affect the rate of sphincter-sparing surgery in rectal cancer? Surg Oncol 2012;21(3):e103-e109

17 Heald RJ, Moran BJ, Ryall RD, Sexton R, MacFarlane JK. Rectal cancer: the Basingstoke experience of total mesorectal excision, 1978-1997. Arch Surg 1998;133(8):894-899

18 Martling A, Holm T, Rutqvist LE, et al. Impact of a surgical training programme on rectal cancer outcomes in Stockholm. Br J Surg 2005;92(2):225-229

19 Ricciardi R, Virnig BA, Madoff RD, Rothenberger DA, Baxter NN. The status of radical proctectomy and sphincter-sparing surgery in the United States. Dis Colon Rectum 2007;50(8):1119-1127, discussion 1126-1127

20 Ito M, Sugito M, Kobayashi A, Nishizawa Y, Tsunoda Y, Saito N. Relationship between multiple numbers of stapler firings during rectal division and anastomotic leakage after laparoscopic rectal resection. Int J Colorectal Dis 2008;23(7):703-707

21 Kim JS, Cho SY, Min BS, Kim NK. Risk factors for anastomotic leakage after laparoscopic intracorporeal colorectal anastomosis with a double stapling technique. J Am Coll Surg 2009;209(6): 694-701

22 Ruo L, Guillem JG. Major 20th-century advancements in the management of rectal cancer. Dis Colon Rectum 1999;42(5): 563-578

23 Marks JH, Frenkel JL, D’Andrea AP, Greenleaf CE. Maximizing rectal cancer results: TEM and TATA techniques to expand sphincter preservation. Surg Oncol Clin N Am 2011;20(3):501-520, viii-ix

24 Di Palo S, De Nardi P, Chiari D, Gazzetta P, Staudacher C. Laparoscopic TME with APPEAR (Anterior and Perineal PlanE for ultralow Anterior Resection of the Rectum) technique for distal rectal cancer. Surg Endosc 2013;27(9):3430

25 Rasheed S, Bowley DM, Aziz O, et al. Can depth of tumour invasion predict lymph node positivity in patients undergoing resection for early rectal cancer? A comparative study between T1 and T2 cancers. Colorectal Dis 2008;10(3):231-238

26 Nash GM, Weiser MR, Guillem JG, et al. Long-term survival after transanal excision of T1 rectal cancer. Dis Colon Rectum 2009; 52(4):577-582

27 Bhangu A, Brown G, Nicholls RJ, Wong J, Darzi A, Tekkis P. Survival outcome of local excision versus radical resection of colon or rectal carcinoma: a Surveillance, Epidemiology, and End Results (SEER) 
population-based study. Ann Surg 2013;258(4):563-569, discussion 569-571

28 Perez RO, Habr-Gama A, Lynn PB, et al. Transanal endoscopic microsurgery for residual rectal cancer (ypT0-2) following neoadjuvant chemoradiation therapy: another word of caution. Dis Colon Rectum 2013;56(1):6-13

29 Lamarque PL, Gros CG. La radioth'erapie de contact des cancers du rectum. J RadiolElectrol 1946;27:333-348

30 Christoforidis D, McNally MP, Jarosek SL, Madoff RD, Finne CO. Endocavitary contact radiation therapy for ultrasonographically staged T1 NO and T2 N0 rectal cancer. Br J Surg 2009;96(4): 430-436
31 Dietz DW; Consortium for Optimizing Surgical Treatment of Rectal Cancer (OSTRiCh). Multidisciplinary management of rectal cancer: the OSTRICH. J Gastrointest Surg 2013;17(10):1863-1868

32 Nougaret S, Rouanet P, Molinari N, et al. MR volumetric measurement of low rectal cancer helps predict tumor response and outcome after combined chemotherapy and radiation therapy. Radiology 2012;263(2):409-418

33 Burton S, Brown G, Daniels IR, Norman AR, Mason B, Cunningham D; Royal Marsden Hospital, Colorectal Cancer Network. MRI directed multidisciplinary team preoperative treatment strategy: the way to eliminate positive circumferential margins? Br J Cancer 2006;94(3):351-357 